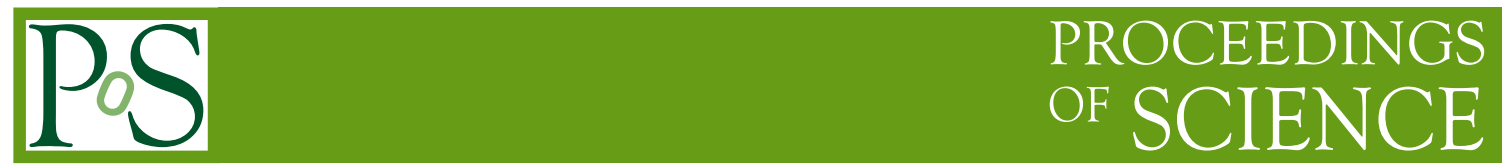

\title{
Playing cards as a tool to create public interest in physics
}

\author{
Jason Veatch* and Yuko Veatch \\ Georg-August-Universität Göttingen, \\ Göttingen, Germany \\ E-mail: jason.veatch@cern.ch, qedplayingcards@gmail.com
}

A novel outreach project is presented that makes use of playing cards - one of the most ubiquitous toys around the world - to communicate physics in a fun, engaging manner. A custom deck of cards has been designed to inspire an interest in physics while being widely appealing to the general public and useful for gameplay, magic and cardistry.

The European Physical Society Conference on High Energy Physics 26-30 July 2021

Online

${ }^{*}$ Speaker 
The QUANTUM deck of playing cards is a standard 52-card deck, but with added custom features that make it a useful tool for physics outreach and education. The face cards depict luminaries of quantum mechanics, such as Niels Bohr, Max Planck and Marie Curie as shown in Figure 1. It is possible to identify each scientist by the detailed iconography on the card related to their lives and scientific work. A booklet, which is available as a free download, provides a short biography of each physicist along with suggested further reading.

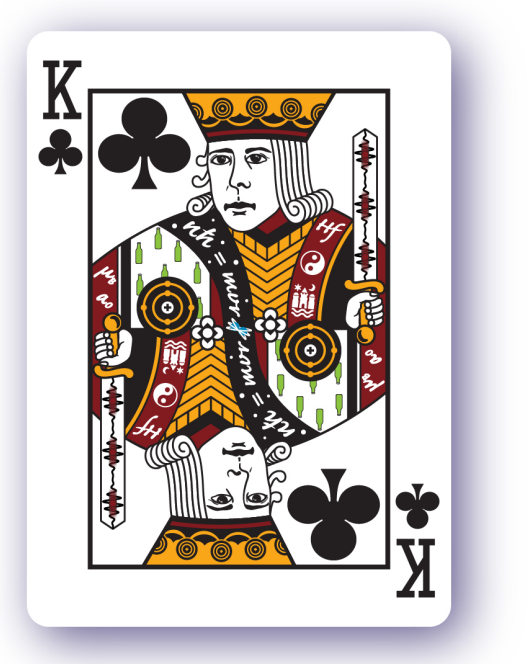

(a)

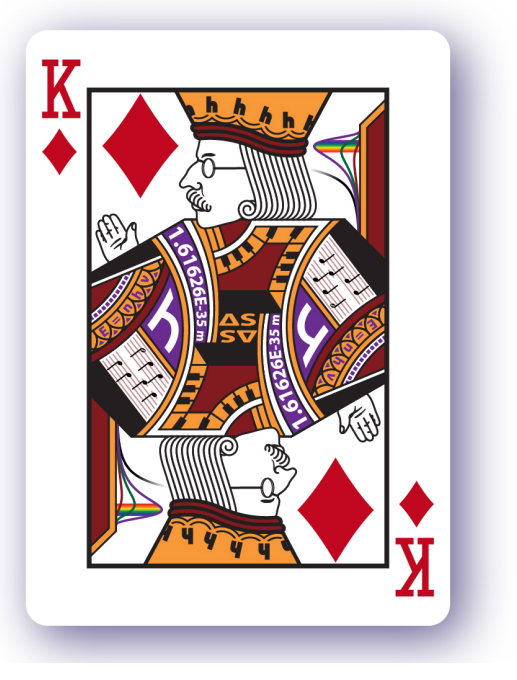

(b)

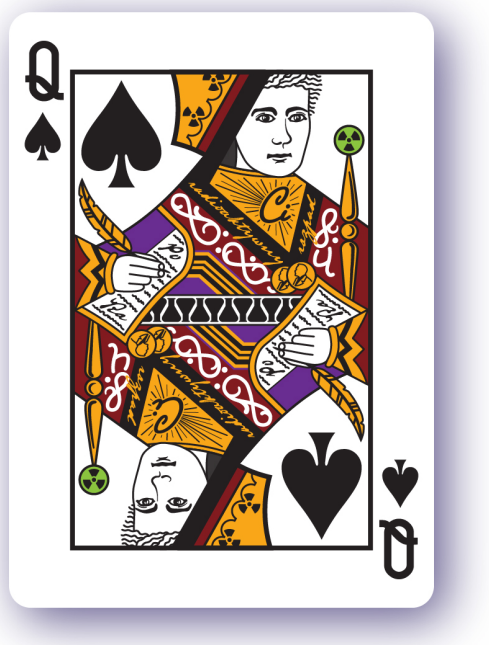

(c)

Figure 1: QUANTUM face cards depicting a Niels Bohr, b Max Planck, and c Marie Curie.

The joker cards depict Schrödinger's cat in the "alive" and "dead" states as shown in Figure 2.

An additional card, shown in Figure 3 provides a detailed explanation of Thomas Young's double-slit experiment as well as instructions and a template that allows individuals to perform the experiment at home. Figure 4 shows an interference pattern created using a laser pointer and the experiment card template. 


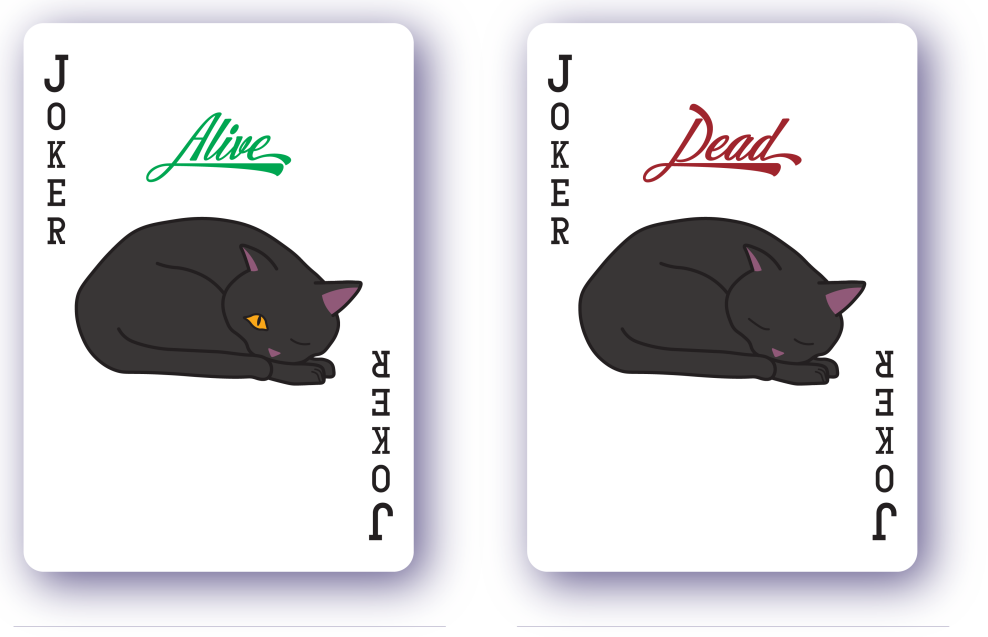

Figure 2: Jokers in the QUANTUM deck depicting "alive” and "dead" states of Schrödinger's cat.
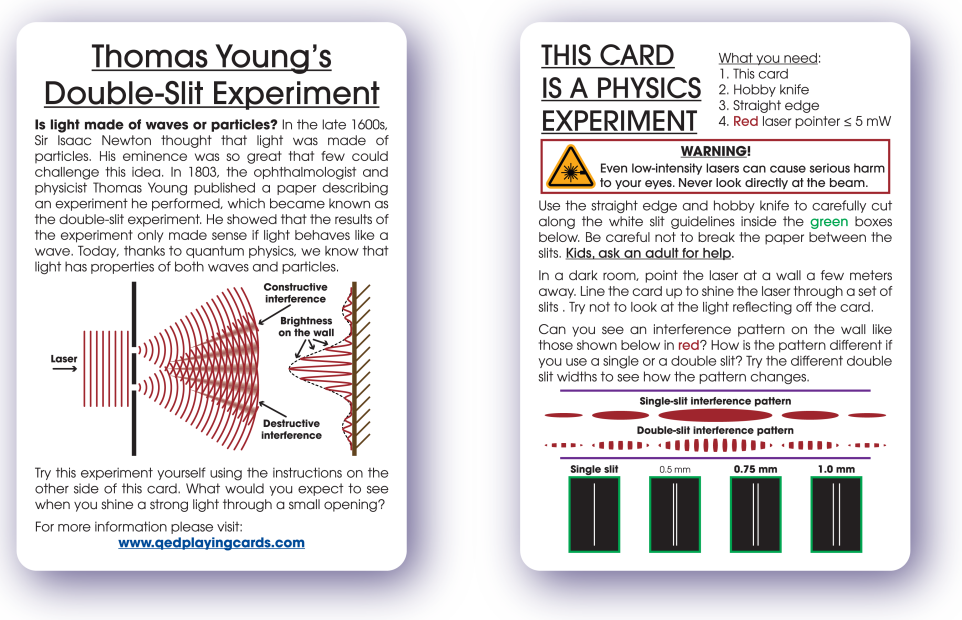

Figure 3: Card with details about how to perform Thomas Young's double-slit experiment at home.

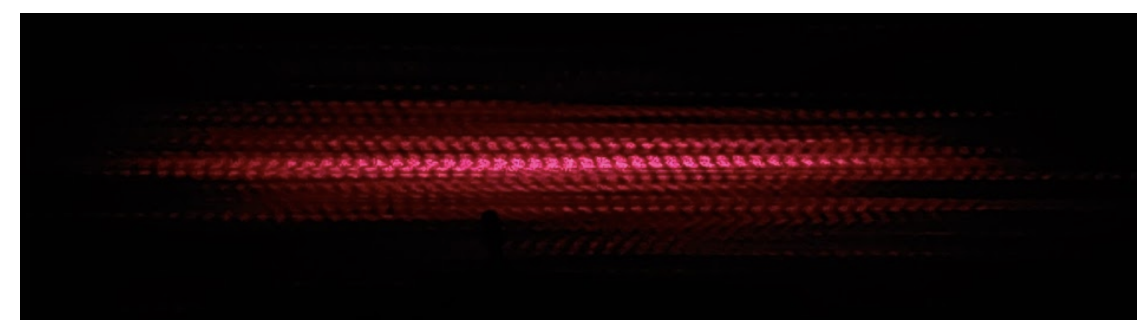

Figure 4: Example of the interference pattern created using a laser pointer and the experiment card template. 
In the course of bringing this project to completion, many different social media platforms and channels have been used to facilitate communication about the project and physics in general. The project has presented opportunities for unusual collaborations and the cards are being used in outreach events and educational settings.

Crowdsourcing was used to raise the funds, obtaining over $400 \%$ of the goal amount. The cards have been printed on high-quality stock by one of the top card printers in the world (Cartamundi). They are available for purchase online and to a limited degree, at brick-and-mortar specialty shops. Special pricing is available for educational and outreach purposes. 